\title{
A phase III study of atezolizumab (atezo) vs placebo as adjuvant therapy in renal cell carcinoma (RCC) patients (pts) at high risk of recurrence following resection (IMmotion010).
}

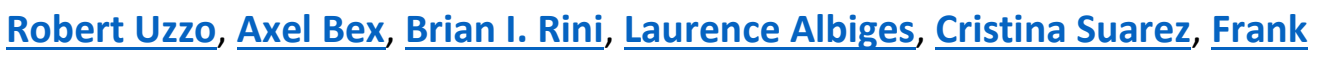

Donaldson, Takashi Asakawa, Christina Schiff, Sumanta K. PalFox Chase Cancer Center, Philadelphia, PA; The Netherlands Cancer Institute, Antoni van Leeuwenhoek Hospital, Amsterdam, Netherlands; Cleveland Clinic Taussig Cancer Insitute, Cleveland, OH; Institut Gustave Roussy, Villejuif, France; Vall d'Hebron University Hospital Institute of Oncology (VHIO), Barcelona, Spain; F. Hoffmann-La Roche Ltd., Basel, Switzerland; Genentech, Inc., San Francisco, CA; City of Hope Comprehensive Cancer Center, Duarte, CAShow Less Abstract Disclosures

\section{- Abstract}

\section{TPS4598}

Background: Nephrectomy is the SOC in early RCC; however, the 5-y relapse rate is $30-40 \%$ in stage II or III pts, with tumor stage and grade correlating with survival and recurrence after surgery. Currently, there is a limited role for adjuvant therapy after nephrectomy in pts who have had complete tumor resection; observation is standard. In a Ph II first-line metastatic RCC study, treatment with single-agent atezo (anti-PD-L1) resulted in an ORR of 25\%. Thus, IMmotion010, a Ph III, multicenter, randomized, placebo-controlled, doubleblinded trial, will evaluate the efficacy and safety of atezo as adjuvant therapy in RCC pts who are at high risk of recurrence after resection (NCT03024996). Methods: Eligible RCC pts (clear cell or sarcomatoid histologies) will have undergone nephrectomy (radical or partial) and be at high risk of recurrence (T2 Grade 4, T3a Grade 3-4, T3b/c any Grade, T4 any Grade or TxN+ any Grade) or have had complete resection of limited metachronous/synchronous metastasis. Pts must show no residual disease or evidence of metastases by CT scan at enrollment. ECOG PS $\leq 1$ and tumor specimens evaluable for PD-L1 will also be required. Pts will be randomized 1:1 to receive atezo $1200 \mathrm{mg}$ IV q3w or placebo IV q3w for 16 cycles or 1 $\mathrm{y}$; stratification will be by disease stage (T2/T3a vs T3b/c/T4/N+ vs metastasectomy), region (North America [excluding Mexico] vs rest of world) and PD-L1 status on tumor-infiltrating immune cells (IC; PD-L1 IC expression $<1 \%$ vs $\geq 1 \%$ ). The primary endpoint is independent review facility (IRF)-assessed disease-free survival (DFS), defined as the time from randomization to the first documented recurrence event (local recurrence, new primary RCC, distant metastasis) or death. Secondary endpoints include OS, investigator-assessed DFS, IRF-assessed and investigator-assessed DFS in pts with $\geq 1 \%$ PD-L1 IC, disease-specific survival, distant metastasis-free survival and the 3-y rates of IRF-assessed DFS and investigator-assessed DFS. Safety and biomarkers will be evaluated. The planned analysis will occur when at least $\approx 65 \%$ of pts in the 2 populations have died. 664 pts will be enrolled at 150-200 sites worldwide. Clinical trial information: NCT03024996. 